\title{
Development of Visual Size Constancy During the 1st Year of Human Infancy
}

\author{
B. E. McKenzie and H. E. Tootell \\ La Trobe University, Bundoora, Victoria, Australia \\ R. H. Day \\ Monash University, Clayton, Victoria, Australia
}

\begin{abstract}
Visual size constancy for distances up to $70 \mathrm{~cm}$ was studied in three experiments with 4-, 6-, and 8-month-old infants and up to $200 \mathrm{~cm}$ with 6-month-old infants in a fourth. A habituation-test procedure was used throughout. At each age subjects were repeatedly shown a three-dimensional model of a human head until a criterion of habituation of looking was reached. Relative to the habituation condition, the standard test condition was either the same (control) or different in distance, size, or both size and distance. Appropriate comparisons between the recovery scores for the test conditions showed that at 6 and 8 months, size constancy occurred for the head model up to a distance of $70 \mathrm{~cm}$. This was not so for $100 \mathrm{~cm}$ and $200 \mathrm{~cm}$. At 4 months size constancy measured in the same way as for older subjects was not apparent in the range $30-60 \mathrm{~cm}$, but there was a suggestion that it is present at this age among those infants with lower variance of responding.
\end{abstract}

Perceptual constancy is the tendency for perceived environmental features to remain relatively stable despite variations in their representation at the sense organs. In a recent critical review of experiments on perceptual constancy in infancy, Day and McKenzie (1977) distinguished between two classes, egocentric and object constancies. The first refers to stability of perceived position, orientation, and movement of objects as their sensory representations change with the posture of the observer, and the second, to stability of perceived intrinsic properties of objects such as their shape, rigidity, brightness, and color as their sensory representations change. Visual size constancy belongs in the latter category. It is the tendency for perceived object size to remain relatively stable as the size of the retinal image varies with changes in observer-object distance.

The assistance of the Australian Research Grants Committee in funding this program is gratefully acknowledged.

Requests for reprints should be sent to R. H. Day, Department of Psychology, Monash University, Clayton, Victoria 3168, Australia.
Four studies concerned with visual size constancy in the 1st year have been reported so far. In two early investigations (Cruikshank, 1941; Misumi, 1951), constancy was indexed by reaching for objects whose size and distance were varied. Unfortunately, the outcomes of both experiments are ambiguous in that they relate as much to the effects of perceived distance as they do to those of perceived size. The only firm conclusion to be drawn from these experiments is that frequency of reaching is not determined solely by the retinal size of the object. This conclusion does not necessarily entail visual size constancy, since reaching for more distant objects might be inhibited regardless of their perceived size.

Two recent studies have sought mainly to detect the existence of size constancy at a particular stage of infancy, usually in the first few months, rather than to chart its development over an extended period. Bower (1974) concluded from his experiments involving generalization of conditioned head turning that visual size constancy is present during the first few months of infancy. The results reported by McKen- 
zie and Day (1972) failed to confirm this conclusion; using recovery of visual fixation and rate of habituation as indices of size constancy, they found no evidence for its occurrence during the period of 6-20 weeks.

In summary, the index of size constancy used in the earlier experiments by Cruikshank (1941) and Misumi (1951) resulted in equivocal findings, and the outcomes of the more recent experiments by Bower (1974) and McKenzie and Day (1972) are inconsistent. Thus no firm conclusions can yet be reached about the occurrence of visual size constancy in the 1st year.

The principal aim of the experiments reported here was to resolve this issue and to chart the development of visual size constancy during the 1 st year. A subsidiary aim was to ascertain the effect on size constancy of the type of stimulus object and its distance from the infant. The strategy adopted was that of first investigating size constancy with 6-month-old babies and, according to the outcome of this experiment, investigating it in either younger or older babies. The main features of the experiments were degree of recovery of visual fixation in standard test trials as an index of size constancy, the use of "interesting" stimulus objects in the form of model human heads, and relatively short viewing distances known to be well within the infant's range of visual attention for stationary objects.

The experimental design was based on the assumption that following habituation to a stimulus object, the magnitude of response recovery is directly related to the magnitude of the perceived change in the most perceptually salient stimulus property. Thus if object size were salient and object distance not so, it would be expected that response recovery to the former would be markedly greater than that to the latter. Therefore, using a habituation-recovery paradigm, size constancy would be indicated when recovery following a change in distance is less than that following a change in physical size or changes in both physical size and distance. The four experiments were predicated on this expectation.

In the first experiment two sizes of otherwise identical model heads were presented at two distances. In the second experiment the same stimulus objects, distances, and procedures were used with 4-month-old infants. In the third experiment with 8-monthold infants, distances were greater by a few centimeters. The fourth experiment involved only 6-month-old infants and was concerned with whether the results for the first experiment would hold at a greater viewing distance and with patterned geometrical objects.

Experimenter reliability was assessed in Experiments 2, 3, and 4, but because of apparatus limitations, it was not established in Experiment 1. However, the high degree of reliability for three experiments justifies the assumption that it was also high in the first.

\section{Experiment 1}

The purpose of the first experiment was to establish whether, in terms of recovery of visual fixation, visual size constancy is evident at 6 months. There were four conditions of habituation to a predetermined criterion: the large head at $60 \mathrm{~cm}$, the large head at $30 \mathrm{~cm}$, the small head at $60 \mathrm{~cm}$, and the small head at $30 \mathrm{~cm}$. The test condition for all four habituation conditions was the large head at $60 \mathrm{~cm}$. For convenience the large head at $60 \mathrm{~cm}$ is designated the control (Co), since the test was identical to it; the large head at $30 \mathrm{~cm}$ the size constancy (SC) condition, since only test size was the same; the small head at $60 \mathrm{~cm}$ the distance (Di) condition, since only test distance was the same; and the small head at $30 \mathrm{~cm}$ the visual angle (VA) condition, since only the visual subtense remained the same.

The logic of the experimental design was as follows. If perceptual constancy is operative at 6 months, it would be expected that recovery of response to the object of the same size at the greater distance (SC) would be most similar to the control (Co) and recovery to the object of a different size (Di and VA) would be greater than the control. If on the other hand, perceived size is determined by the projected size of the object, it would be expected that recovery to the larger object at the greater distance (VA) would be most similar to the control (Co) and that recovery to the larger object at the 
same distance (Di) and the same object at the greater distance (SC) would be greater.

To ascertain whether size constancy occurred, three contrasts were planned (Roger, 1967). The mean recovery score for the SC condition was compared with that for Co, for Di was compared with that for VA, and for SC and Co combined was compared with that for Di and VA combined. The last of these was the most critical; if size constancy occurred, it was expected that the mean of SC and Co together would be less than that of $\mathrm{Di}$ and VA together. However, it was of interest nevertheless to ascertain whether the score for SC was the same or different from that of Co. Of course, it was not necessary that the mean for SC should be the same as that for Co, only that the two together should be less than the other two (Di and VA) together. It was also of interest to ascertain whether the means for Di and VA were different. It seemed conceivable that since Di involved a change in distance only and VA a change in size and distance, the latter would give rise to greater recovery than the former. It is emphasized that the most critical comparison for establishing the occurrence of size constancy was that of the combined scores of SC and Co with those of Di and VA. The other two contrasts were of incidental interest. While other groups of comparisons could have been made, we felt that the three chosen were of greatest relevance for establishing the presence of size constancy. Constraints placed on the number of comparisons by the available degrees of freedom (Roger, 1967) rendered further comparisons questionable. The choice of contrasts is discussed further below.

\section{Method}

Subjects. The sample consisted of 32 infants, 18 males and 14 females, with a mean age of 6 months, ranging from 5.2 months to 7 months. Data from 5 additional infants were not included in the final analysis; 3 infants cried and could not be pacified, and 2 moved excessively, so visual fixation and object distance were difficult to assess. Subjects were solicited from nearby infant health centers and were brought to the laboratory for testing. Only healthy full-term infants were included in the sample. Eight subjects were allocated at random to each of the four experimental conditions described below.
Habituation and test objects. The object chosen to convey information about physical size, projected size, and distance was a colored model of an adult female head. The test model was life-size and was presented at a distance of $60 \mathrm{~cm}$, subtending a visual angle of nearly $24^{\circ}$. The habituation model was either the test model itself or one reduced by half and was presented at either $30 \mathrm{~cm}$ or $60 \mathrm{~cm}$.

Apparatus and procedure. Subjects were placed in an infant seat located in front of a screen $122 \mathrm{~cm} \times$ $117 \mathrm{~cm}$. The gray screen was supported on casters so that it could be moved readily and quietly when viewing distance was changed. The model heads were placed on a hinged support behind the screen and were swung upward, thus activating a microswitch connected to a computer. In position, the heads rested on top of the screen and were viewed against a plain white wall. An experimenter concealed behind the screen viewed the subject through a small aperture and indicated visual fixation by pressing a button. Each object was presented until the look-away time equaled $1 \mathrm{sec}$. The computer indicated the end of a trial with an auditory signal received by the experimenter through headphones. The experimenter removed the model by swinging it downward and then waited until the infant was looking straight ahead before beginning the next trial.

The distance of the subject from the stimulus object was videotaped by a camera positioned on the subject's left. This provided a clear profile view of both infant and stimulus object.

There were four phases in the experiment: pretest, habituation, test, and posttest. A colored ball was used during the pretest and posttest phases to check the level of attentiveness over the session. The test stimulus was always the large model at a distance of $60 \mathrm{~cm}$. The habituation stimuli were the large model at a distance of $30 \mathrm{~cm}$ for the $\mathrm{SC}$ group, the small model at $30 \mathrm{~cm}$ for the VA group, the small model at $60 \mathrm{~cm}$ for the Di group, and the large model at $60 \mathrm{~cm}$ for the Co group. To make the change from the last habituation trial to the test trial in the control condition as similar as possible to those in the other conditions, the screen was moved forward and backward and the stimulus object. was swung down and up. Thus the manipulations and accompanying sounds were more or less the same as for other conditions.

The first trial was the pretest with the colored ball. The habituation trials that followed involved repeated presentation of the appropriate object until the predetermined criterion of habituation was reached or until 18 habituation trials were completed. Only data obtained from subjects who attained the criterion were used in the analysis. The criterion was defined as two consecutive trials on which the looking time was less than half of the mean of the looking time for the first three trials in the habituation phase. The test stimulus was presented on the next two trials. The last trial constituted the posttest with the control stimulus. The end of each trial and the change from one experimental phase to another was signaled by the computer, which also supplied a printout of the frequency and duration of fixation.

The score used throughout to index recovery from habituation during the test phase was the difference 
between the mean of the last two habituation trials and the first test trial. This is referred to as the recovery or difference score.

\section{Results and Discussion}

The mean durations of visual fixation for the pretest and posttest, for the last five habituation trials, and for the first test trial are shown in Figure 1. The points for the pretest and posttest are based on 32 subjects, whereas those for the habituation trials and the test trial are based on 8 subjects.

The results were analyzed in terms of the level of attentiveness over the session as indicated by a comparison between the pretest and posttest trials; of the similarity of the experimental groups, by a comparison between the means of the first three habituation trials and between the number of trials to criterion; and of the recovery of visual fixation for the four groups, by a comparison of the difference scores between the mean of the last two habituation trials and the first test trial.

Pretest and posttest trials. Change in the level of attention over the experimental session was assessed by comparing fixation time for the control stimulus (the colored ball) on the first and last trials. A twoway analysis of variance with trials (2) as a within-subjects factor and experimental condition (4) as a between-subjects factor showed no significant main effects and no interaction. That is, fixation of the control stimulus did not decline after habituation and test trials for any of the groups.

Habituation trials. The mean fixation time for the first three habituation trials was $9.7 \mathrm{sec}$ and the mean number of trials to criterion was 10.5. Two separate oneway analyses of variance were carried out to compare the initial level of fixation, as measured by the mean of the first three habituation trials, and the number of trials required to attain the habituation criterion for the four experimental groups. The groups did not differ significantly on either measure. Thus it can be assumed that the stimulus objects were not grossly different in attractiveness prior to habituation treatment, and the relative habituation criterion was attained by each group at approximately the same rate.

Test trials. Since the distribution of difference scores was nonhomogeneous, logarithmic transformations were carried out and the analysis performed on the trans-

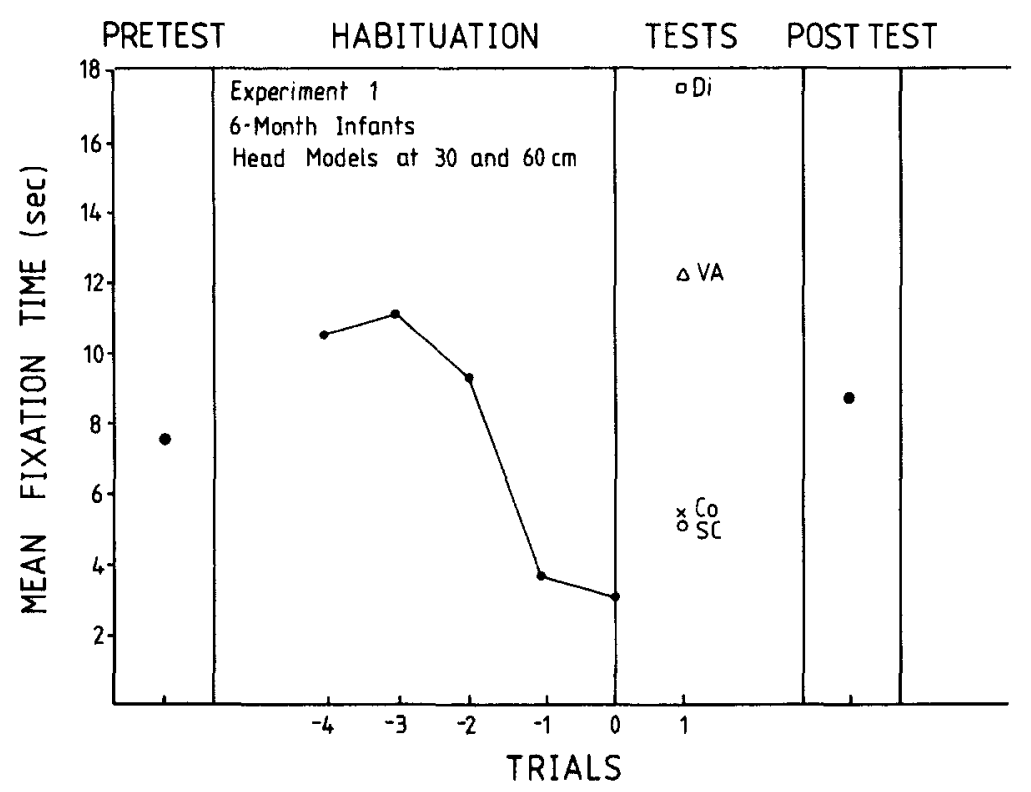

Figure 1. Mean fixation times for pretest and posttest trials, last five habituation trials, and four test trials in Experiment 1. (The abbreviations VA, Di, SC, and Co refer respectively to the visual angle, distance, size constancy, and control conditions of the test phase.) 
formed scores. The means of these scores for the SC, VA, Di, and Co groups were .16, $.96,1.0$, and .67 , respectively. The first contrast showed that the mean recovery score for the SC group was not different from that of the Co group, and the second, that the difference between the VA and the Di groups was likewise not significant. In the third contrast the mean of the combined $\mathrm{SC}$ and Co groups was compared with that of the combined VA and Di groups. The latter was significantly greater than the former, $F(1,28)=9.01, p<.01$. Thus the increase in looking time following a change in object distance was not different from that of the control condition, and that following a change in size was not different from that following a change in size and distance; but the changes for these last two considered together exceeded those for the control and distance changes considered together.

Since prior to habituation the experimental groups did not differ in fixation times for the different stimuli and since the test stimulus was the same for each group, differential increments in recovery scores during the test phase can be confidently attributed to the habituation treatment. The same series of planned contrasts performed on the absolute, rather than the transformed, scores led to the same conclusion. There was no difference between the means of the SC and the Co groups, no difference between the means of the VA and the Di groups, but the mean of the combined $\mathrm{SC}$ and Co groups was significantly less than that of the combined VA and Di groups, $F(1,28)=6.96, p<.05$. In addition, the recovery on the first test trial was maintained on the second trial, since the means for the two did not differ.

In summary, the groups did not differ in the prehabituation levels of attention or in the rate of attainment of the criterion of habituation. The degree of response recovery associated with a change in object distance was much less than that associated with a change in physical size and changes in both physical size and distance, suggesting that size constancy is operative with this age group. Whether this is so for 4month-old infants was examined in the next experiment.

\section{Experiment 2}

\section{Method}

Subjects. The sample was comprised of 32 infants, 17 males and 15 females, with a mean age of 4.2 months, ranging from 3.7 to 4.5 months. Six infants who cried and a further 5 who did not reach the habituation criterion were replaced.

Apparatus and procedure. The apparatus and procedure were the same as in Experiment 1, except that a second camera was introduced in order to record visual fixation on the stimulus object. This camera was positioned behind and above the screen and was focused on the infant's eyes.

\section{Results and Discussion}

Reliability. Since the observer was aware of the stimulus conditions while observing fixation, an independent measure of observer reliability was included in this and subsequent experiments. This was achieved through an analysis of videotaped records by a scorer who was ignorant of the experimental treatment. Look-away times were measured on the records. Errors were of two types, those in which the look-away time exceeded $1 \mathrm{sec}$ before the trial was terminated and those in which the time was less than $1 \mathrm{sec}$ when the trial was terminated. Only those trials associated with the difference scores, that is, the last two habituation trials and the first test trial, were considered. The error rate on these trials was $5.6 \%$. Neither the type nor the frequency of error was clearly associated with the experimental treatment. Thus it seems unlikely that experimenter bias or unreliability of recording distorted the outcome of the experiment.

Pretest and posttest, habituation, and test scores. Mean fixation times for the pretest, and posttest, the last five habituation trials, and the first test trial are shown in Figure 2. There was no significant difference in duration of fixation for the pretest and posttest trials. Neither the mean of the first three habituation trials nor the number of trials required to reach the habituation criterion differed between the experimental groups. The four groups began by looking for approximately the same amount of time and habituated at about the same rate. It can be noted that the mean fixation time for 


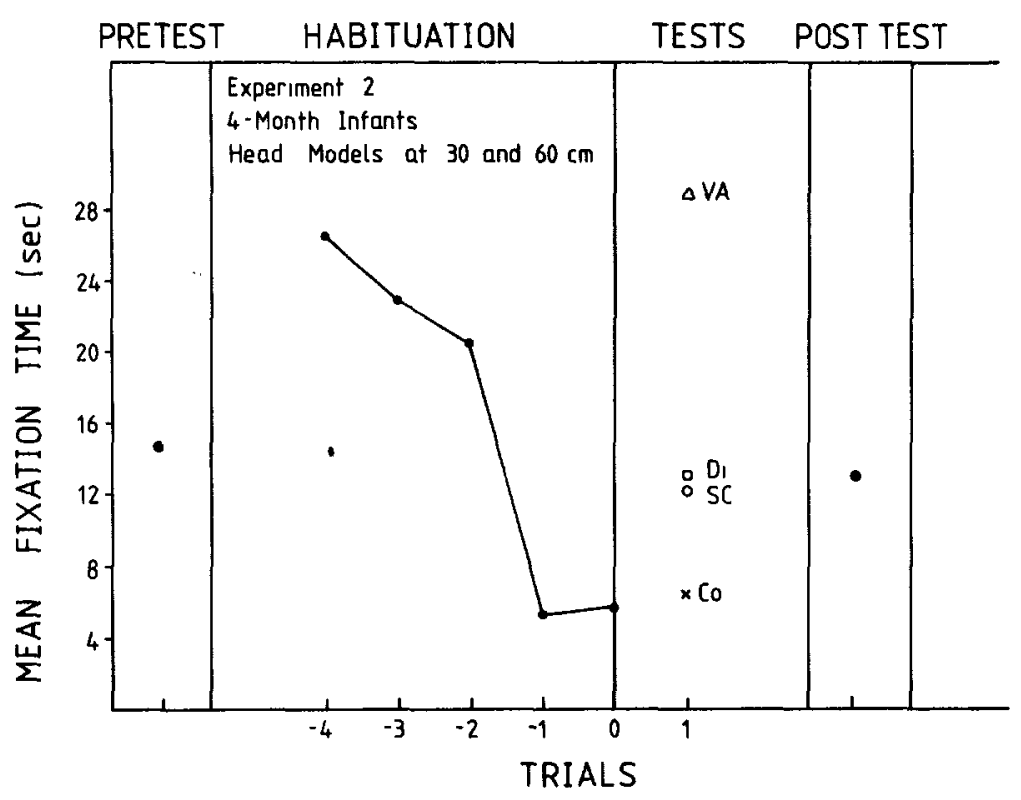

Figure 2. Mean fixation times for pretest and posttest trials, last five habituation trials, and four test trials in Experiment 2. (The abbreviations VA, Di, SC, and Co refer respectively to the visual angle, distance, size constancy, and control conditions of the test phase.)

the three habituation trials $(27.2 \mathrm{sec})$ was notably longer than that $(9.7 \mathrm{sec})$ for the 6month-old group, whereas the mean number of trials to criterion was not markedly different, 8.5 as compared with 10.5 .

The same planned contrasts as before were used to analyze the logarithmically transformed recovery scores. The means for the SC, VA, Di, and Co groups were 1.09, $1.15,1.24$, and .85 , respectively. The mean for the SC group was not different from that for the $\mathrm{Co}$, the mean score for the VA group was not different from that for the Di group, and the mean for the combined SC and Co groups was not different from that for the combined VA and Di groups. Thus, none of the contrasts was reliable, although the mean of the last two habituation trials was less than the mean of the first test trial, $F(1,28)=6.42, p<.05$. That is, although there was a recovery of visual fixation, the degree of recovery was independent of the habituation treatment. Analysis of the untransformed scores led to the same conclusion; none of the three contrasts was reliable. Recovery on the first test trial was not maintained at the same level on the second test trial, $F(1,28)=4.98, p<.05$.
Thus, whereas the results of Experiment 1 suggested that by 6 months size constancy is operating, those of Experiment 2 provide no evidence that this is so at 4 months. At the earlier age all changes-distance, physical size, physical size and distance, and the control manipulations - were equally effective in producing recovery of visual fixation.

An incidental observation noted in the course of the experiment was that the variability of looking for the 4-month-old subjects was greater than that for the 6-month-old subjects in Experiment 1. It was conceivable that infants with lower variability might show a pattern of recovery different from those with higher variability. For this reason the variance of fixation duration in the habituation trials was calculated for each subject. The total group was then split at the median of the variance so that there were 16 infants whose looking time was less variable. This split produced 6 subjects from the SC group, 3 from the VA group, 6 from the Di group, and 1 from the Co group. The transformed mean difference scores for these groups were $.43,1.2, .87$, and .16 , respectively, whereas for the more variable 
group, these means were $1.33, .82,1.37$, and .84 , respectively. It can be observed that the means of the former group approximate the pattern of magnitudes obtained for the 6-month-old infants in the first experiment and those of the latter group do not. It seems reasonable to suggest, therefore, that at about 4 months size constancy might be operative for those infants whose variability in looking time is least and most similar to that of older infants. This tentative suggestion will be taken up again in the Discussion section.

In the third experiment the prediction that 8-month-old infants would respond in the same manner as the 6-month-old group was tested.

\section{Experiment 3}

\section{Method}

Subjects. There were 32 subjects from the same source as in the earlier experiments. Of these 15 were males and 17 females. The mean age was 7.8 months, ranging from 6.5 to 8.7 months. The results of 15 subjects were not included in the final analysis because of problems with behavioral state and because 1 infant did not reach the habituation criterion. It can be noted that relative to 4 - and 6-month-old infants, a markedly greater number in this age group displayed distress in the experimental situation.

Apparatus and procedure. The same head models, apparatus, and procedures were used, except that the distances were increased to $35 \mathrm{~cm}$ and $70 \mathrm{~cm}$ so that infants' feet were not touching the screen as they would have done at $30 \mathrm{~cm}$.

\section{Results and Discussion}

Reliability. Look-away times were scored from the videotaped records as in Experiment 2. The error rate for the last two habituation trials and the first test trial was $1.6 \%$, notably less than in the second experiment with younger subjects. Since so few errors occurred and since neither type nor frequency of errors was associated with particular experimental conditions, it is reasonable to suppose that the recovery scores were not unduly affected by bias or unreliability of recording.

Pretest and posttest, habituation, and test scores. Mean fixation times for the pretest and posttest trials, the last five habituation trials, and the first test trial are shown in Figure 3. The mean transformed difference scores for the SC, VA, Di, and Co groups were $1.07,1.22,1.34$, and $.98 \mathrm{sec}$, respectively. The difference between the

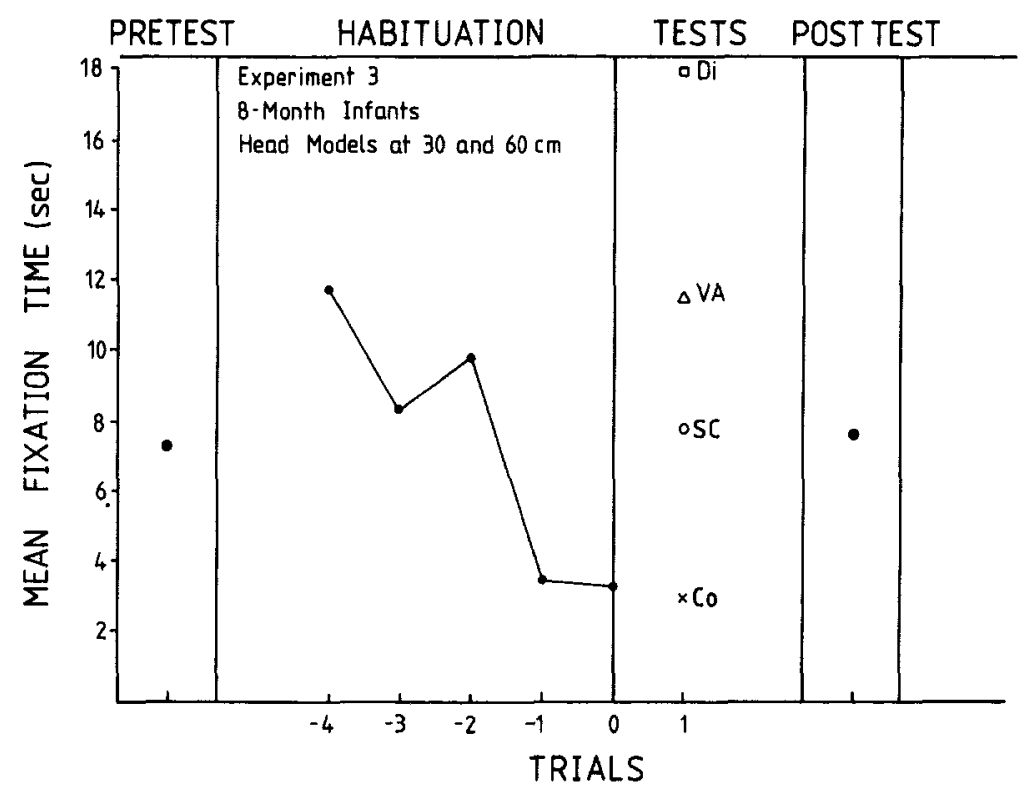

Figure 3. Mean fixation times for pretest and posttest trials, last five habituation trials, and four test trials in Experiment 3. (The abbreviations VA, Di, SC, and Co refer respectively to the visual angle, distance, size constancy, and control conditions of the test phase.) 
mean pretest and posttest fixation times proved not to be significant. Neither the mean fixation time of the first three habituation trials nor the mean number of trials to reach criterion differed significantly between groups. The mean of the former was $11.3 \mathrm{sec}$, and that of the latter was 7.8. The planned contrasts showed no difference in the transformed mean difference scores between the SC and the Co groups or between the VA and Di groups. The mean for the combined SC and Co groups was significantly less than that for the combined VA and Di groups, $F(1,28)=$ $26.18, p<.01$. Similar results were obtained with the untransformed scores; only the mean differences for the combined groups attained significance, $F(1,28)=$ $15.28, p<.01$. The recovery scores on Test Trial 2 were less than those for Test Trial 1 , $F(1,28)=6.5, p<.05$. The mean fixation time on Test 1 was $10.0 \mathrm{sec}$, and on Test 2 , $6.9 \mathrm{sec}$.

These results are essentially the same as those for the 6-month-old subjects in Experiment 1 in showing that the degree of recovery of looking with change in distance alone is no different from that of the control, whereas the recoveries with changes in size and in size and distance are greater. That is, the outcomes are in accord with what can be expected if visual size constancy is operative.

\section{Experiment 4}

The restricted extent of the effective visual field of young infants has now been amply confirmed in a number of recent studies (Aslin \& Salapatek, 1975; de Schonen, McKenzie, Maury, \& Bresson, 1978; Macfarlane, Harris, \& Barnes, 1976). Most studies have involved infants younger than 2 months, and the effective field has been plotted by using the occurrence and latency of visual saccades in the direction of the target. McKenzie and Day (1972) observed that even 5-month-old infants rarely looked at a patterned cube at a distance of $90 \mathrm{~cm}$ regardless of its projected size at the eye. However, if the object were moving, the effective visual field was greatly extended (McKenzie \& Day, 1976). Although objects may be detected in more distant space, they may still present difficulties for higher perceptual processing (de Schonen et al., 1978). In this case, size constancy might be characteristic of near, but not far, objects. It is also conceivable that it is characteristic of familiar relevant objects rather than of unfamiliar irrelevant objects. Both possibilities were investigated in the fourth experiment.

\section{Method}

The same design and procedure were used for this experiment as for the three earlier ones, but the distances were increased to $100 \mathrm{~cm}$ and $200 \mathrm{~cm}$. Infants were tested on two occasions, once with model heads and once with patterned cubes, with the order counterbalanced over subjects.

Subjects. A further sample of 32 6-month-old infants was drawn from the same population. Data from 3 other subjects were excluded from the analysis because of equipment failure or because of failure by subjects to attain the habituation criterion. Infants were assigned at random to one of the four experimental conditions. The mean age was 6.2 months, with a range of 5.8-6.8 months. The average time between the first and second testing was 1 week.

Apparatus and procedure. The same models of heads were used, as well as cubes measuring $26 \mathrm{~cm}$ and $13 \mathrm{~cm}$ on a side and patterned with black and white checks proportional to the size of the cube. As before, the colored ball was used for the pretests and posttests. The test object was always the large head or large cube at $200 \mathrm{~cm}$. The stimulus object conditions for the habituation trials corresponded essentially with those in the earlier experiments. At the end of each trial, curtains were closed and then reopened with the appropriate stimulus in place for the beginning of the next trial. The opening of the curtains operated a microswitch signaling the beginning of the trial to the computer. Apart from these minor changes, the procedure was identical to that of the earlier experiments.

\section{Results and Discussion}

Reliability. With heads as stimulus objects, there was a total of 3 errors $(2.4 \%)$ during the last two habituation trials and the first test trial, and with cubes, a total of 4 (3.4\%). Again the type and frequency of error were not associated with particular conditions. Thus it can be concluded that neither observer bias nor unreliability of recording affected the outcomes of the experiment. 
Pretest and posttest, habituation, and test scores. Mean fixation times for the pretest and posttest trials, the last five habituation trials, and first test trial are shown for the head models in the top portion of Figure 4 and for the cubes in the bottom portion of Figure 4. The mean fixation time for the first three habituation trials was 11.1 sec for heads and $4.4 \mathrm{sec}$ for cubes. The mean number of trials to criterion when heads were presented was 7.1 , and when cubes were presented, 6.8. The mean transformed difference scores for the SC, $\mathrm{VA}, \mathrm{Di}$, and Co groups were $1.18,1.11$, 1.27 , and .96 , respectively, for the heads and $1.1,1.06,1.01$, and 1.96 , respectively, for the cubes.

With the head models as habituation and test stimuli, there was a significant difference in looking times for the pretest and posttest trials, $F(1,28)=8.99, p<.01$. However, since this difference represents

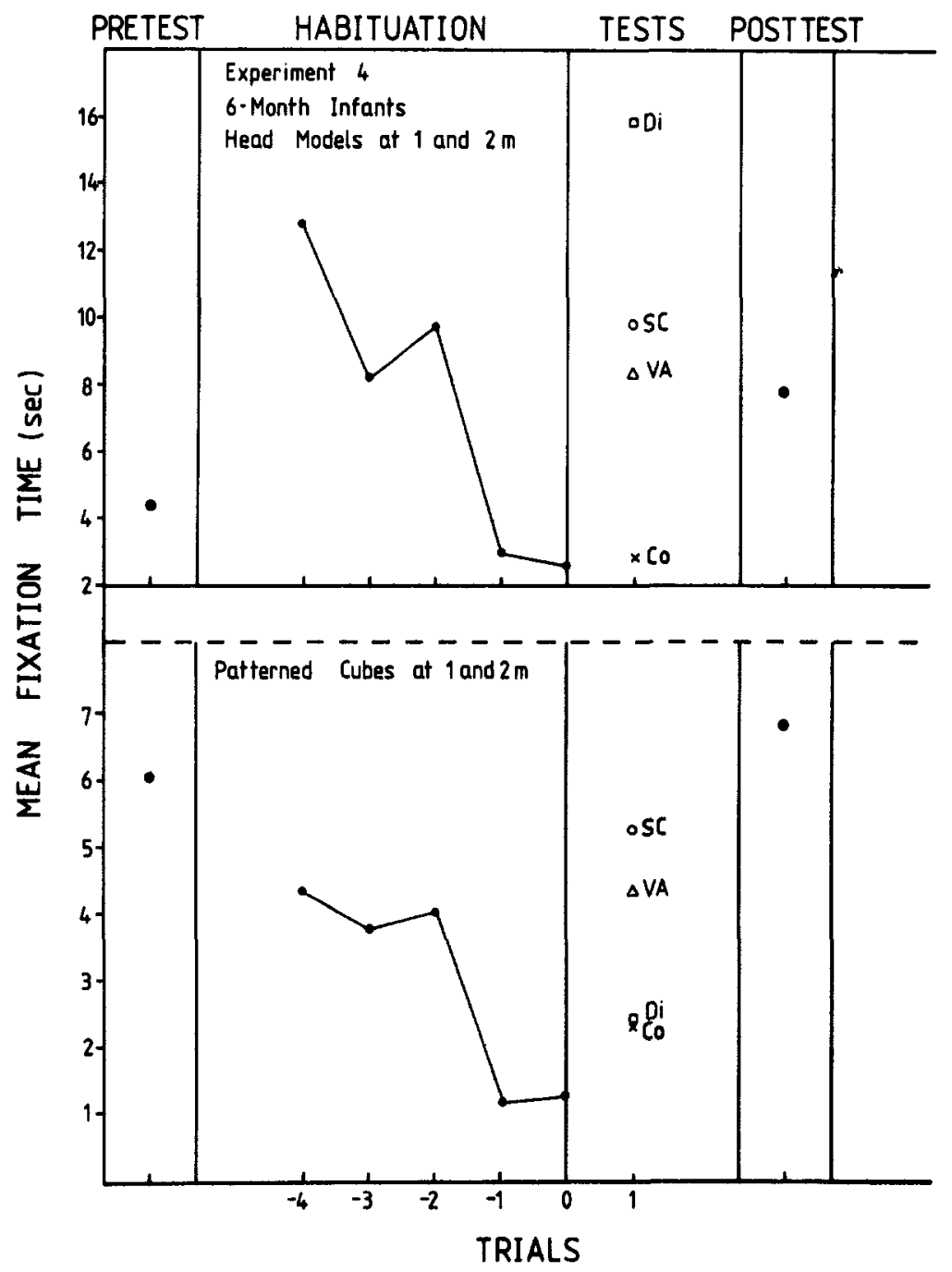

Figure 4. Mean fixation times for pretest and posttest trials, last five habituation trials, and four test trials in Experiment 4 for two stimulus objects, head models and patterned cubes. (The abbreviations VA, Di, $\mathrm{SC}$, and Co refer respectively to the visual angle, distance, size constancy, and control conditions of the test phase.) 
an increase on the last trial, it can be assumed that attention was not declining over the session in a nonspecific manner. Neither the mean for the first three habituation trials nor the number of trials to reach criterion differed significantly between groups. Despite the apparent difference in recovery scores (Figure 4), none of the planned contrasts was significant for either transformed or untransformed scores. In addition, there was no significant difference between the first and second test trials.

Using the cubes as habituation and test stimuli, there was no overall difference in fixation times for the pretest and posttest trials. There was a significant difference in the mean fixation time for the first three habituation trials between groups, $F(3,21)=$ $4.1, p<.05$. This did not appear to be a systematic effect of the size of the cubes, but rather of the distance of presentation. The mean looking times over the first three habituation trials for cubes were $4.0 \mathrm{sec}$ at $100 \mathrm{~cm}$ and $4.8 \mathrm{sec}$ at $200 \mathrm{~cm}$. There was no difference between the groups in the number of trials to reach criterion. None of the planned contrasts comparing the mean differences in transformed or untransformed recovery scores were significant; that is, recovery was not related to the habituation treatment. Fixation times decreased on the second test trial, $F(1,28)=$ $4.78, p<.01$. In summary, the pattern of results for recovery of response after habituation did not accord with expectation for perceptual constancy of size for either the head models or for the patterned cubes.

\section{Discussion}

In the four experiments described here, the standard test condition following habituation under four conditions represented a change in distance with size constant (SC), a change in size with distance constant (Di), a change in size and distance with visual angle constant (VA), or no change at all (Co). The following comparisons were made between the recovery scores for the four conditions: between Co and SC, between Di and VA, and between Co and SC combined and Di and VA combined. The last of these was regarded throughout as the main index of the operation of size constancy. In the experiments with 6- and 8month-old infants using interesting stimulus objects in the range $30-70 \mathrm{~cm}$, the scores for $\mathrm{Co}$ and SC combined were less than those for Di and VA combined. This was not so in the experiment with 4-month-old infants or in that with both model heads and cubes located at $100 \mathrm{~cm}$ and $200 \mathrm{~cm}$. In all experiments neither the difference between Co and SC nor that between Di and VA was significant.

Considered together these data are taken to indicate that size constancy is operative for infants of 6 and 8 months observing interesting objects that are relatively nearup to $70 \mathrm{~cm}$-but not for objects much beyond that range.

Although this conclusion has been reached largely by comparing the combined recovery scores for the Co and SC conditions with those for the $\mathrm{Di}$ and VA conditions, the comparison between $\mathrm{Co}$ and $\mathrm{SC}$ also favors the conclusion. In all experiments this difference failed to reach significance. For 4-month-old infants, it was one of three differences that did not reach significance, but at 6 and 8 months, when the difference between Co plus SC and Di plus VA achieved significance, it continued to be insignificant. In other words, at a stage when change in object size alone and object size and distance together were selectively responded to, a change in visual angle with size constant was not responded to. Such selective responding as indexed by recovery scores strongly suggests that whereas changes in object size and size and distance together elicit marked attention, a change in distance with object size constant does not. It can be noted that had one of the planned contrasts been between SC and Di plus VA, the conclusion for each experiment would have been no different.

It is also of interest to note that throughout the experiments the differences between recovery scores for conditions $\mathrm{Di}$ and VA were not significant. It seemed possible at the beginning of the experiments that changing two features of the stimulus array (size and distance as in VA) would give rise 
to a greater recovery from habituation than a change in size alone (as in Di). That this was not so can be interpreted in various ways: The identity of visual angle in VA may have counteracted the effect of change in two features of the array, or alternatively, a change in size may have been salient so that a simultaneous change in distance might not therefore have been attended to. There are other possible interpretations. However, this is a side issue that, although worthy of further inquiry, cannot be settled on the basis of the data reported here.

The recovery scores for 4-month-old infants were divided into two groups, that for which the variance in habituation trials was below the median and that for which it was above. The pattern of recovery score magnitudes of the subgroup with lower variance was similar to that of the older infants, whereas that of the other subgroup was not. This originally unplanned division of the scores for the 4-month-old infants suggests that size constancy, as indexed by the relative magnitude of recovery scores for the four conditions, is beginning to become apparent at 4 months among those subjects whose variability of response is relatively low. This view must be treated as tentative and suggestive for the time being. It is put forward here as a basis for further experiments rather than as a firm conclusion.

Of course comparisons between conditions or combinations of conditions other than those chosen could have been made. However, the number of orthogonal contrasts among the four conditions was necessarily restricted to three by the requirements of statistical logic (Roger, 1967). An alternative comparison relevant to the constancy hypothesis is that between conditions SC and VA. Both conditions involved a change in distance; however, the second involved a change in object size, whereas the first did not. Had this contrast been planned and carried out, the conclusions reached here would not have been altered. A further analysis showed that although the difference between the scores for SC and VA were not significant for 4-month-old infants, recovery in condition VA was significantly greater than in SC for both 6- and 8-month-old infants. While an additional contrast is questionable on statistical grounds, the result is in accord with the contrasts already made. Thus had alternative comparisons been made, the conclusions reached would have been the same.

The general conclusion to be drawn from these experiments is that infants in the range 6-8 months and, presumably, older infants appear to attend predominantly to changes in object size rather than to changes in distance. In terms of recovery from habituation, this selectivity of attention occurred spontaneously for the older infants, but not for the younger infants. However, the pattern of scores for some members of the younger group is suggestive of the presence of size constancy. Of course it is possible that stronger evidence for the occurrence of size constancy, or at least for the presence of a potential for it, might emerge using alternative methods. For example, such might be the case using a variant of the habituation procedure, in which an object is presented repeatedly at numerous distances and then in the test, the same object and one of a different size but within the range of visual angles for habituation are presented. It is also possible that training of the sort involved in reinforcement procedures would produce such evidence. What can be said on the basis of the results reported here and summarized in Figures 1-4 is that using the habituationrecovery method with interesting relatively near objects, there is good evidence for the operation of size constancy from 6 months of age and older and a suggestion that it is present at around 4 months.

\section{References}

Aslin, R. N., \& Salapatek, P. Saccadic localization of visual targets by the very young human infant. Perception \& Psychophysics, 1975, 17, 293-302.

Bower, T. G. R. Development in infancy. San Francisco: Freeman, 1974.

Cruikshank, R. M. The development of visual size constancy in early infancy. Journal of Genetic Psychology, 1941, 58, 327-351.

Day, R. H., \& McKenzie, B. E. Constancies in the perceptual world of the infant. In W. Epstein (Ed.), Stability and constancy in visual perception: Mechanisms and processes. New York: Wiley, 1977. 
de Schonen, S., McKenzie, B. E., Maury, L., \& Bresson, F. Central and peripheral object distances as determinants of the effective visual field in early infancy. Perception, 1978, 7, 499-506.

Macfarlane, A., Harris, P., \& Barnes, I. Central and peripheral vision in early infancy. Journal of Experimental Child Psychology, 1976, 21, 532-538.

McKenzie, B. E., \& Day, R. H. Object distance as a determinant of visual fixation in early infancy. Science, 1972, 178, 1108-1110.

McKenzie, B. E., \& Day, R. H. Infant's attention to stationary and moving objects at different distances. Australian Journal of Psychology, 1976, 28, 45-51.

Misumi, J. Experimental studies on the development of visual size constancy in early infancy. Bulletin of the Faculty of Literature (Kyushu University), $1951,1,91-116$.

Roger, R. S. Type I errors and their decision basis. British Journal of Mathematical and Statistical Psychology, 1967, 20, 51-62.

Received August 23, 1979

\section{Acknowledgment}

In addition to the staff of regular consulting editors, other scholars are often asked to help in reviewing manuscripts submitted to the Journal. The Editor wishes to acknowledge with thanks the advice and consultation provided by the following persons who served recently as guest editors.

\author{
Eugene Abravanel \\ Jack Arbuthnot \\ Frank R. Ascione \\ Terry R. Barrett \\ David J. Bearison \\ John N. Bohannon \\ Inge Bretherton \\ Donna Cohen \\ Phebe Cramer \\ Nancy W. Denney \\ Francine Deutsch \\ Helen J. Emmerich \\ Catherine J. Garvey \\ Margaret J. Gatz \\ Rochel S. Gelman \\ Herbert P. Ginsburg \\ Joseph Glick \\ Gerald Gratch \\ William G. Graziano \\ Esther B. Greif \\ Merrill Hiscock \\ Albert R. Hollenbeck \\ John C. Houtz \\ David F. Hultsch \\ David Klahr
}

Leon Kuczynski

Lawrence A. Kurdek

Michael E. Lamb

Michael Lewis

Raymond P. Lorion

Michael M. Marcell

Daphne Maurer

Robert B. McCall

Curtis W. McIntyre

Richard L. Metzger

Scott A. Miller

Barbara E. Moely

James W. Pellegrino

John M. Polich

Hayne W. Reese

Diane N. Ruble

John M. Rybash

Carolyn I. Saarni

Timothy A. Salthouse

Eli Saltz

Frances F. Schachter

Jan M. D. Sinnott

Colleen Surber

Judith L. Whatley

Elizabeth Zelinski 